\title{
Significant Inhibition of Tumor Growth following Single Dose Nanoparticle-Enhanced Photodynamic Therapy
}

\author{
Shih-Hsun Cheng, ${ }^{1,2}$ Chia-Hui Chu, ${ }^{1}$ Nai-Tzu Chen, ${ }^{1,2}$ Jeffrey S. Souris, ${ }^{2}$ \\ Chin-Tu Chen, ${ }^{2}$ and Leu-Wei Lo ${ }^{1}$ \\ ${ }^{1}$ Institute of Biomedical Engineering and Nanomedicine, National Health Research Institutes, Zhunan, \\ Miaoli County 35053, Taiwan \\ ${ }^{2}$ Department of Radiology, The University of Chicago, Chicago, IL 60637, USA
}

Correspondence should be addressed to Leu-Wei Lo; lwlo@nhri.org.tw

Received 25 February 2014; Accepted 1 June 2014; Published 7 August 2014

Academic Editor: Quan-Guang Zhang

Copyright ( 2014 Shih-Hsun Cheng et al. This is an open access article distributed under the Creative Commons Attribution License, which permits unrestricted use, distribution, and reproduction in any medium, provided the original work is properly cited.

\begin{abstract}
Photodynamic therapy (PDT) for cancer treatment involves the pathology's uptake of photosensitizers, which produce cytotoxic reactive oxygen species by photoirradiation. The use of nanoparticles as carriers of photosensitizers is one promising approach to this endeavor, owing to their small size, unique physicochemical properties, and easy/diverse functionalization. In the current work, we report on the in vivo assessment of PDT efficacy of these nanoconstructs in a murine model of human breast cancer, following a single (one-shot) nanoparticle dose and photoirradiation. Palladium-porphyrin (PdTPP) was administered intratumorally via injection of aqueous suspensions of either free PdTPP or MSN-conjugated PdTPP (MSN-PdTPP) at a dose of $50 \mu$ g. Mice were then exposed to a single photoirradiation session with total energy of $80 \mathrm{~J}$. One month after one-shot PDT treatment, significantly greater reductions in tumor growth were observed in MSN-Pd treated animals than in PdTPP cohorts. Electron microscopy of tumor specimens harvested at various timepoints revealed excellent MSN-PdTPP uptake by cancer cells while immunohistologic analysis demonstrated marked increases in apoptotic response of MSN-PdTPP treated animals relative to PdTPP controls. Taken together, these findings suggest that considerable improvements in PDT efficacy can readily be achieved via the use of nanoparticlebased photosensitizers.
\end{abstract}

\section{Introduction}

Photodynamic therapy (PDT) has evolved considerably in recent years to treat a number of medical conditions that include wet macular degeneration, dermatologic disorders (e.g., acne, rosacea, and psoriasis), and several types of cancer including lung and esophageal cancer [1-6]. It is intrinsically a focal therapy far less invasive than surgical approaches and with fewer adverse effects than systemic chemotherapy. The most prevalent forms of PDT in use today are based on the photogeneration of cytotoxic, reactive oxygen species within tumor tissue via the optical stimulation of exogenous photosensitizing agents (PSs). The photosensitizer, in its ground (singlet) state, absorbs a photon that promotes the PS to a short-lived excited singlet state. Because the excited singlet state is so short-lived, the PS has little opportunity to transfer either energy or electrons to other molecules nearby and instead undergoes intersystem crossing to leave it in an excited, much longer-lived, triplet state. As the PS subsequently decays back to its initial ground (singlet) state, it transfers energy to nearby ground (triplet) state molecular oxygen, raising the latter to its first excited singlet state $[7,8]$. Most photosensitizers in clinical use have been optimized/selected for their high, excited singlet state quantum efficiency and recursively generate substantial amounts of singlet oxygen from molecular oxygen rather than undergoing thermal decay (via internal conversion) or fluorescent emission $[9,10]$.

The most widely employed PSs at present are porphyrins. Photoirradiation of porphyrins at selective wavelengths of visible light $(450-700 \mathrm{~nm})$ leads to the photochemical conversion of molecular oxygen $\left({ }^{3} \mathrm{O}_{2}\right)$ into singlet oxygen $\left({ }^{1} \mathrm{O}_{2}\right)$, 


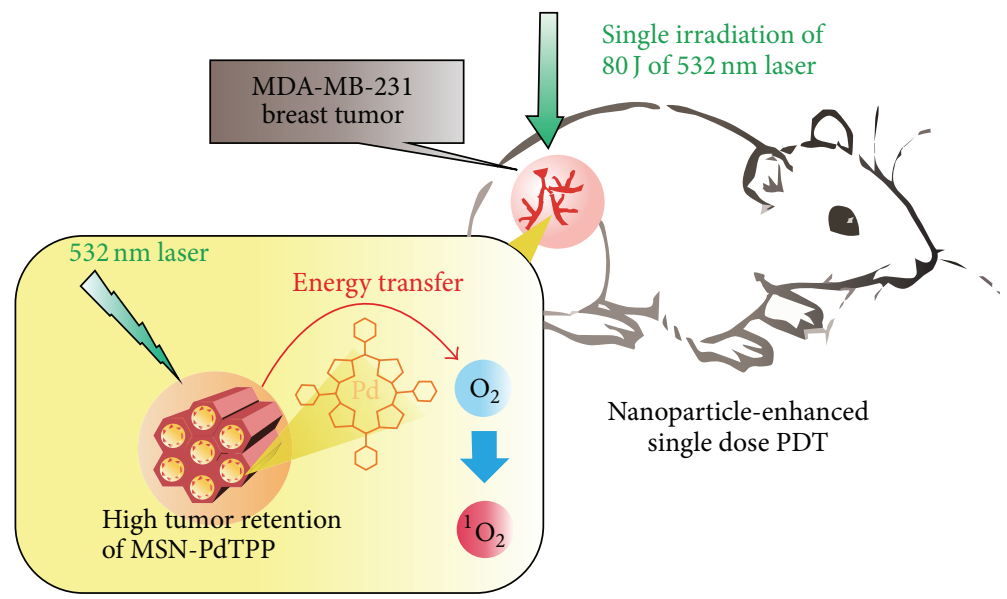

Scheme 1: The illustration of in vivo PDT studies using MSN-PdTPP with single photoirradiation on a murine model bearing human breast cancer cells.

an especially efficacious cytotoxic agent that leads to apoptosis or necrosis [11]. In the conventional PDT of cancer, a photosensitizer drug is administered to the patient, which then passively accumulates in the tumor tissue, though a few PSs have intrinsic proclivities for targeting the endothelial cells of vasculature. The involved tissue is then selectively illuminated with light which activates the photosensitizer inducing cell death. Photosensitizers have evolved over the years to maximize their potency and simultaneously minimize their systemic toxicity. Unfortunately, to date, the overwhelming majority of photosensitizers still possess multiple limitations that include high hydrophobicity, significant selfaggregation, and poor tumor selectivity, each limiting PDT efficiency and clinical benefit $[12,13]$. As such, considerable effort has gone into developing compounds with improved "deliverability."

One approach to enhancing PS delivery is that of nanoparticle- (NP-) mediated transport. When compared to conventional unshielded delivery, NP-assisted conveyance of PSs has the added advantage of providing an exoplatform for the conjugation of polymers to prolong PS circulation halflives and thereby increase PS accumulation in the leaky vasculature of tumors via the enhanced permeability and retention effect (EPR) [14-19]. Encapsulation of PSs within NPs also circumvents PS self-aggregation and enables high density PS delivery. NP conveyance of PSs also permits the incorporation of efficient, light-absorbing chromophores in close proximity to PSs, so as to greatly promote the energy transfer to PSs and thereby significantly enhance overall photodynamic efficiency [20-26]. One form of nanoparticles in particular has recently gained considerable interest for use both in cancer chemotherapy and in PDT: mesoporous silica nanoparticles (MSNs) [27-32].

MSNs are biocompatible, readily endocytosed, and easily modified after synthesis for the conjugation of targeting moieties on their outer surfaces [33-39]. The unique topology of MSNs also provides them with three distinct domains that can be independently functionalized: the silica framework, the hexagonal nanochannels/pores, and the nanoparticle's outermost surface. As such, MSNs are especially well suited to the task of incorporating the essential capabilities of a combined diagnostic/therapeutic (i.e., theranostic) platform in a single particle, with (1) separate domains for contrast agents that enable traceable imaging of PS targeting, (2) PS payloads for therapeutic intervention, and (3) biomolecular ligands for highly targeted PS delivery.

Previously, we reported the development and in vitro characterization of MSNs that were sequentially functionalized with the fluorescent contrast agent ATTO647N embedded within the MSN's silica framework for tracking, the cRGDyK peptide conjugated onto the MSN's exterior for targeting the $\alpha_{v} \beta_{3}$ integrin expression, and the photosensitizer Pd-porphyrin (PdTPP) covalently linked within the MSN's protective nanochannels for photodynamic therapy [40-42]. Herein we report the results of our in vivo PDT studies of MSNs-PdTPP using a murine model of human breast cancer and one-shot photoexcitation (Scheme 1).

\section{Methods}

2.1. Materials. Tetraethoxysilane (TEOS), cetyltrimethylammonium bromide (CTAB), ethanol, ammonium hydroxide (30\%), N,N-dimethylformamide (DMF), and 3-Aminopropyltrimethoxysilane (APTMS) were purchased directly from Acros. Di(N-succinimidyl) carbonate (DSC) and N,Ndiisopropylethylamine were obtained from Sigma Chemical Co.

2.2. Preparation of MSNs. The sol-gel co-condensation of TEOS to synthesize MSNs was as follows. First, CTAB (0.58 g) was dissolved in $\mathrm{NH}_{4} \mathrm{OH}(0.51 \mathrm{M}, 300 \mathrm{~mL})$ at $40^{\circ} \mathrm{C}$. After stirring for $1 \mathrm{~h}$, diluted TEOS $(0.2 \mathrm{M}$ in $5 \mathrm{~mL}$ ethanol) was added and the solution was stirred for an additional $4 \mathrm{~h}$. Then, high concentration of TEOS $(1.0 \mathrm{M}$ in $5.0 \mathrm{~mL}$ of ethanol) was added under vigorous stirring for an additional hour. The solution was aged in darkness at $40^{\circ} \mathrm{C}$ for $20 \mathrm{~h}$. Samples were subsequently collected by centrifuging at $12,000 \mathrm{rpm}$ for 
20 min, washing, and redispersing the solids with deionized water and ethanol repeatedly. Surfactant templates were removed by extraction in $0.3 \mathrm{~g}$ of $\mathrm{NH}_{4} \mathrm{NO}_{3}$ and $50 \mathrm{~mL}$ of ethanol solution under reflux at $65^{\circ} \mathrm{C}$ for $12 \mathrm{~h}$.

2.3. Preparation of MSN-PdTPP. As the surfactant templates were being removed to yield MSNs, $50 \mathrm{mg}$ PdTPP and $150 \mathrm{mg}$ di(N-succinimidyl) carbonate (DSC) were mixed with $100 \mu \mathrm{L}$ N,N-diisopropylethylamine (99.5\%) in N,Ndimethylformamide (DMF) $(20 \mathrm{~mL})$ solution for $2 \mathrm{~h}$. The activated PdTPP was then reacted with $200 \mu \mathrm{L}$ APTS for $1 \mathrm{~h}$ and purified by a PD-10 column (Amersham Biosciences). The amount of PdTPP loading in MSNs was determined by measuring the absorbance at $400 \mathrm{~nm}$, the Soret band of PdTPP. The amounts of PdTPP in MSNs were thereby determined to be $5.5 \%$ w.t.

2.4. Characterization. The morphology of MSN samples was characterized via TEM (Hitachi, H-7650), operating at an acceleration voltage of $80 \mathrm{kV}$. Surface areas and pore sizes were determined by $\mathrm{N}_{2}$ adsorption-desorption isotherm measurements at $77 \mathrm{~K}$ on a Micrometric ASAP 2010. Samples were outgassed at $10^{-3}$ Torr and $120^{\circ} \mathrm{C}$ for approximately $6 \mathrm{~h}$ prior to conducting adsorption experiments. Pore size distribution curves were obtained from analysis of the desorption portion of the isotherms using the BJH (BarrettJoyner-Halenda) method. Steady-state absorption spectra were acquired with a DU800 UV spectrometer (Beckman). A Malvern Zetasizer Nano was used to measure the zeta potential of MSN samples in solution at $\mathrm{pH}$ 7.4.

2.5. Singlet Oxygen Measurement. We used a DPBF ( $8 \mathrm{mM})$ acetonitrile solution to measure single oxygen generation. $2 \mathrm{~mL}$ DPBF solution was added to the PdTPP and MSNPdTPP samples and mixed thoroughly. The mixture was then irradiated at $532 \mathrm{~nm}$ via a laser operating at $250 \pm 5 \mathrm{~mW} \mathrm{~cm}^{-2}$. The absorption spectra of DPBF were detected at $400 \mathrm{~nm}$ every 20 seconds.

2.6. Cell Culture. MDA-MB-231 human breast carcinoma cells were maintained in RPMI-1640 medium (Gibco-BRL, NY, USA) with $10 \%$ heat-inactivated fetal bovine serum (FBS) (Hyclone, Utah, USA) and supplemented with 100 units/mL penicillin and $100 \mu \mathrm{g} / \mathrm{mL}$ streptomycin at $37^{\circ} \mathrm{C}$ with $5 \% \mathrm{CO}_{2}$.

2.7. In Vitro PDT. A total of 5000 MDA-MB-231 breast cancer cells were seeded on a 96-well and incubated for $24 \mathrm{~h}$. Cells were then treated with 10,25 , and $50 \mu \mathrm{g} / \mathrm{mL}$ of either PdTPP or MSN-PdTPP in a serum-free medium for $1 \mathrm{~h}$ at $37^{\circ} \mathrm{C}$ in darkness, respectively. Cell viability was estimated by WST-1 assay. In the WST-1 assay, tetrazolium salt WST-1 \{4-[3-(4-lodophenyl)-2-(4-nitrophenyl)2H-5-tetrazolio]-1, 3-benzene disulfonate in live cells was cleaved by mitochondrial dehydrogenase to yield formazan. After treatment for $1 \mathrm{~h}$, the supernatant was removed and added to $100 \mu \mathrm{L} /$ well serum-free medium and $10 \mu \mathrm{L} /$ well Cell Proliferation Reagent WST-1 (Roche). After incubation with
WST-1 for $4 \mathrm{~h}$, plates were shaken for $1 \mathrm{~min}$ and measured for optical absorbance at $450 \mathrm{~nm}$ using an ELISA reader (Infinite M200, TECAN). Six specimens were run for each concentration, with each experiment being repeated three times. For the PDT, cells were irradiated using a $532 \mathrm{~nm}$ laser operating at $250 \pm 5 \mathrm{~mW} \mathrm{~cm}^{-2}$ at energies of $1.2,2.5$, or $5 \mathrm{~J}$ after treatment of either PdTPP or MSN-PdTPP in a serumfree medium for $1 \mathrm{~h}$ at $37^{\circ} \mathrm{C}$.

2.8. Tumor Xenograft Animal Model. MDA-MB-231 human breast carcinoma cells $\left(1 \times 10^{6}\right.$ cells $/ 200 \mu \mathrm{L}$ sterile saline $)$ were subcutaneously injected into the dorsal region of both thighs of male nude mice ( $\mathrm{nu} / \mathrm{nu}$; 20-25 g; 6-8 weeks of age; BioLasco Taiwan Co., Ltd). Tumor growth curves were obtained using daily digital caliper measurement of tumor diameter once the bulge caused by the tumor cells at the injection site became visible (tumor size $\sim 200 \pm 50 \mathrm{~mm}^{3}$ ). Tumor volume $\left(\mathrm{mm}^{3}\right)$ was calculated using the formla: $0.523 \times$ (length $\times$ width $\times$ thickness) and assessed twice per week for one month. All experiments involving animals were performed in accordance with the guidelines of institutional animal care and use committee.

2.9. In Vivo Two-Photon Excited PDT. In vivo PDT experiments were performed when the tumor volume reached approximately $200 \pm 50 \mathrm{~mm}^{3}$. Mice were divided into groups of twelve animals to minimize variations in group weight and tumor size. Tumor-bearing nude mice were anaesthetized and treated by intratumoral injection of PdTPP or MSNPdTPP at a concentration of $50 \mu \mathrm{g}$. One hour after injection, mice were subjected to $80 \mathrm{~J}$ of $532 \mathrm{~nm}$ laser irradiation such that the average power of light delivered to the skin was $235 \mathrm{~mW}$. Control group mice received intratumoral injections of saline with or without irradiation, respectively. Mice were monitored for a maximum of 28 days. The length, width, and thickness of tumors were measured by digital calipers and assessed twice weekly for one month. All mice were euthanized at the end of the 28th day and tumors were removed for further study.

2.10. Tissue Preparation for Histopathology and Immunohistochemistry. At the end of experiments, a thorough necropsy of mice was carried out immediately after euthanasia. Samples of the tumor mass were embedded in OCT (Tissue-Tek) and stored in $-20^{\circ} \mathrm{C}$ freezer. Samples were sectioned at $10 \mu \mathrm{m}$ thickness and then stained with hematoxylin and eosin (H\&E), caspase-3 (abcam), and TUNEL stain (Roche, in situ cell death detection kit) for examination by light microscopy.

For H\&E staining, sections from cryopreserved tissues were fixed in $4 \%$ paraformaldehyde and stained with hematoxylin and eosin. Following staining, sections were dehydrated, mounted, and observed.

For caspase-3 staining, sections from cryopreserved tissues were incubated with $3 \%(\mathrm{v} / \mathrm{v})$ hydrogen peroxide in methanol for $15 \mathrm{~min}$ and in 3\% BSA for $1 \mathrm{~h}$ to block endogenous peroxidases and nonspecific binding. Sections were then incubated with polyclonal antibodies to active caspase3 (1:500) in diluting buffer ( $1 \%$ bovine serum albumin, 


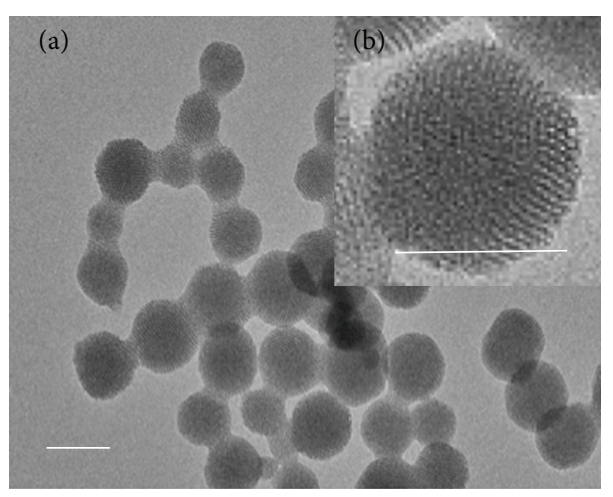

(d)

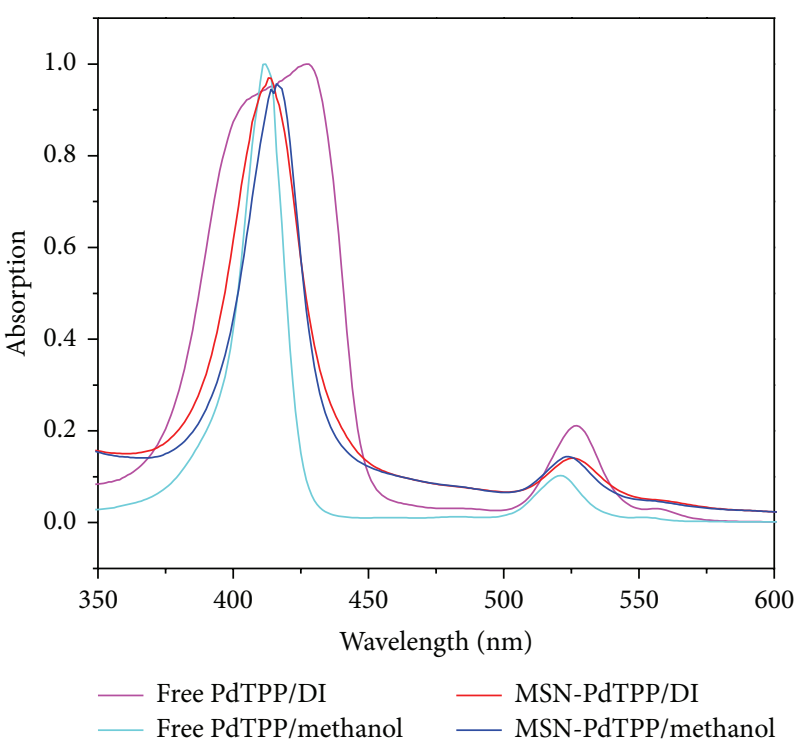

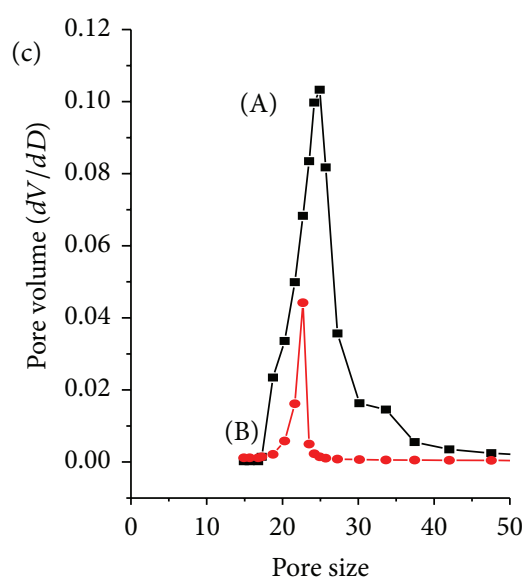

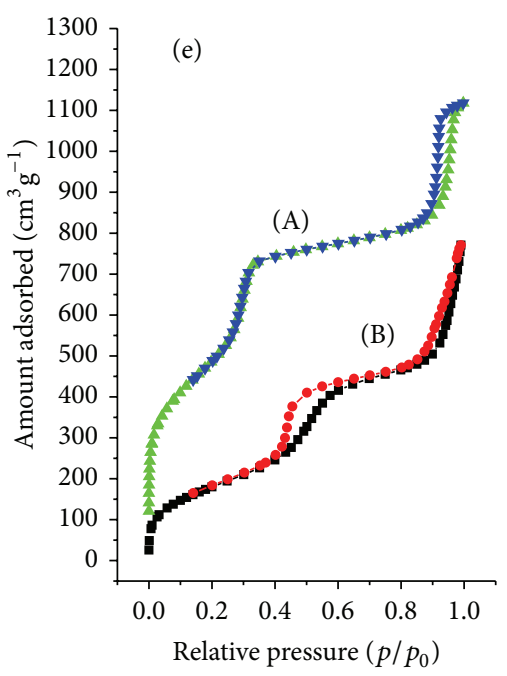

Figure 1: Nanoparticle characterization. (a) TEM images of MSN-PdTPP nanoparticles (scale bar: $100 \mathrm{~nm}$ ). (b) Well-ordered porous structure of MSN-PdTPP (scale bar: $100 \mathrm{~nm}$ ). (c) Pore size distribution of (A) MSN and (B) MSN-PdTPP derived from desorption isotherm measurements and BJH method. (d) UV-visible absorption spectra of PdTPP and MSN-PdTPP in DI water and methanol. (e) Nitrogen adsorption-desorption isotherms of (A) MSN and (B) MSN-PdTPP.

$0.1 \%$ Tween-20 in PBS) overnight at $4^{\circ} \mathrm{C}$. Sections were next incubated with goat anti-rabbit HRP secondary antibodies (Jackson, $1: 10000$ ) in PBS for $1 \mathrm{~h}$. Detection of caspase-3 positive cells was undertaken via Diaminobenzidine (DAB) immunohistochemistry, followed by counterstaining with Gills Haematoxylin.

For TUNEL staining, sections were fixed in $4 \%$ paraformaldehyde and blocked in 3\% hydrogen peroxide in methanol for $15 \mathrm{~min}$. Next specimens were incubated in permeabilization solution $(0.1 \%$ triton $\mathrm{X}-100$ and $0.1 \%$ sodium citrate in PBS) for 2 min on ice. TUNEL reaction mixture was added and incubated for $1 \mathrm{~h}$ at $37^{\circ} \mathrm{C}$ in the dark. After PBS rinsing, samples were incubated with converter-POD for $30 \mathrm{~min}$ at $37^{\circ} \mathrm{C}$. Detection of apoptosed cells was achieved using DAB immunohistochemistry, followed by counterstaining with Gills Haematoxylin.
2.11. Transmission Electron Microscopy Imaging of Tumor Tissue. For electron microscopy, tumor tissue specimens were fixed overnight in glutaraldehyde, buffered (2.5\%) with PBS. Tissues were then washed with 3 changes of PBS and postfixed for $1 \mathrm{~h}$ in a solution containing $\mathrm{OsO}_{4}$ buffered (2\%) with PBS. Samples were next washed in 3 changes of $\mathrm{dH}_{2} \mathrm{O}$ and dehydrated stepwise in ethanol. Tissues were polymerized using Spurr resin at $68^{\circ} \mathrm{C}$ for 15 hours. The embedded specimens were then thin-sectioned at $70 \mathrm{~nm}$ and viewed on a Hitachi H-7650 transmission electron microscope operating at $80 \mathrm{kV}$.

\section{Results and Discussion}

The intrinsic flexibility of MSN surface chemistry readily affords postsynthesis, application-specific modification, for 
example, tailoring for optimal drug release, dispersion stability, cellular uptake, and attachment of imaging agents and targeting ligands. For the current application, the porphyrinbased photosensitizer PdTPP was attached via the addition of amino groups to the MSN's outer surfaces and nanochannel walls. Amino modification of MSN surfaces also served to increase the overall surface charge of the nanoplatform (zeta potential of MSN-PdTPP: $+28.5 \mathrm{mV}$ ), thereby enhancing cell uptake and minimizing self-aggregation (and thus selfquenching). Di(N-succinimidyl) carbonate (DSC) coupling was used to encapsulate PdTPP to the MSNs. MSN-PdTPP morphology was characterized by combined transmission electron microscopy (TEM), $\mathrm{N}_{2}$ adsorption-desorption isotherm measurement, and UV-Vis spectroscopy. TEM studies demonstrated well-ordered, hexagonal porous structures characteristic of mesoporous silica, with average particle diameters of $80 \mathrm{~nm}$ (Figures 1(a) and 1(b)). Surface area and pore size of MSNs and MSN-PdTPPs were determined by $\mathrm{N}_{2}$ adsorption-desorption isotherm measurements (Figures 1(c) and $1(\mathrm{e})$ ). Following PdTPP incorporation, nanoparticle surface areas decreased from $985 \mathrm{~m}^{2} \mathrm{~g}^{-1}$ (MSN) to $632.06 \mathrm{~m}^{2} \mathrm{~g}^{-1}$ (MSN-PdTPP). Measured UV absorption spectra of MSNPdTPP and PdTPP showed MSN conjugation greatly increasing PdTPP solubility in water via possessing only slight differences in methanol solutions but significant differences in DI water (Figure 1(d)) - a consequence of the solubility of PdTPP in water being poor, and its aggregation broadening the Soret band spectra. Quantization of the PdTPP content of MSN conjugates was achieved by measuring each sample's absorbance at $400 \mathrm{~nm}$ (PdTPP Soret band) while dissolved in HF-NaF, averaging 5.5\% PdTPP by weight.

We assessed the generation of singlet oxygen $\left({ }^{1} \mathrm{O}_{2}\right)$ by photoirradiation of MSN-PdTPP in water using an indirect chemical method: one that employed 1,3-diphenylisobenzofuran (DPBF), whose optical absorption at $400 \mathrm{~nm}$ decreases in the presence of singlet oxygen. We also measured the singlet oxygen production of photoirradiated free PdTPP in water for comparison (10.4 $\mu \mathrm{g}$ of PdTPP, in both free form and MSN-conjugated form). Singlet oxygen generation in aqueous suspensions of MSN-PdTPP and free PdTPP, wth $532 \mathrm{~nm}$ laser diode irradiation and $20 \mathrm{sec}$ interval spectra sampling, is shown in Figure 2 as a function of illumination time. Singlet oxygen generation of DPBF-only solutions and DPBF solutions bearing MSNs (data not shown) served as controls. Much steeper reductions were found in the optical absorption of DPBF solutions containing MSN-PdTPP than of nanoparticle-free samples, reflecting the former's significantly higher efficiency in generating ${ }^{1} \mathrm{O}_{2}$.

For the in vitro cytotoxicity analysis of our nanoplatforms, WST-1 assays of MDA-MB-231 cell viability were used following $1 \mathrm{~h}$ incubations with either 10,25 , or $50 \mu \mathrm{g} \mathrm{mL}^{-1}$ of MSN-PdTPP or free PdTPP-both before and after photoirradiation. To assess optimal illumination, we photoirradiated these groups at one of three different total energies (1.5, 2.5 , and $5 \mathrm{~J})$ using a $532 \mathrm{~nm}$ diode-laser $\left(250 \pm 5 \mathrm{~mW} / \mathrm{cm}^{2}\right)$. As shown in Figure 3(b), little photo-induced cytotoxicity was found for $25 \mu \mathrm{g}$ and $50 \mu \mathrm{g}$ free PdTPP treatments, with viabilities of $81 \%$ for $25 \mu \mathrm{g} \mathrm{mL} L^{-1}$ and $80 \%$ for $50 \mu \mathrm{g} \mathrm{mL}^{-1}$ at $5 \mathrm{~J}$

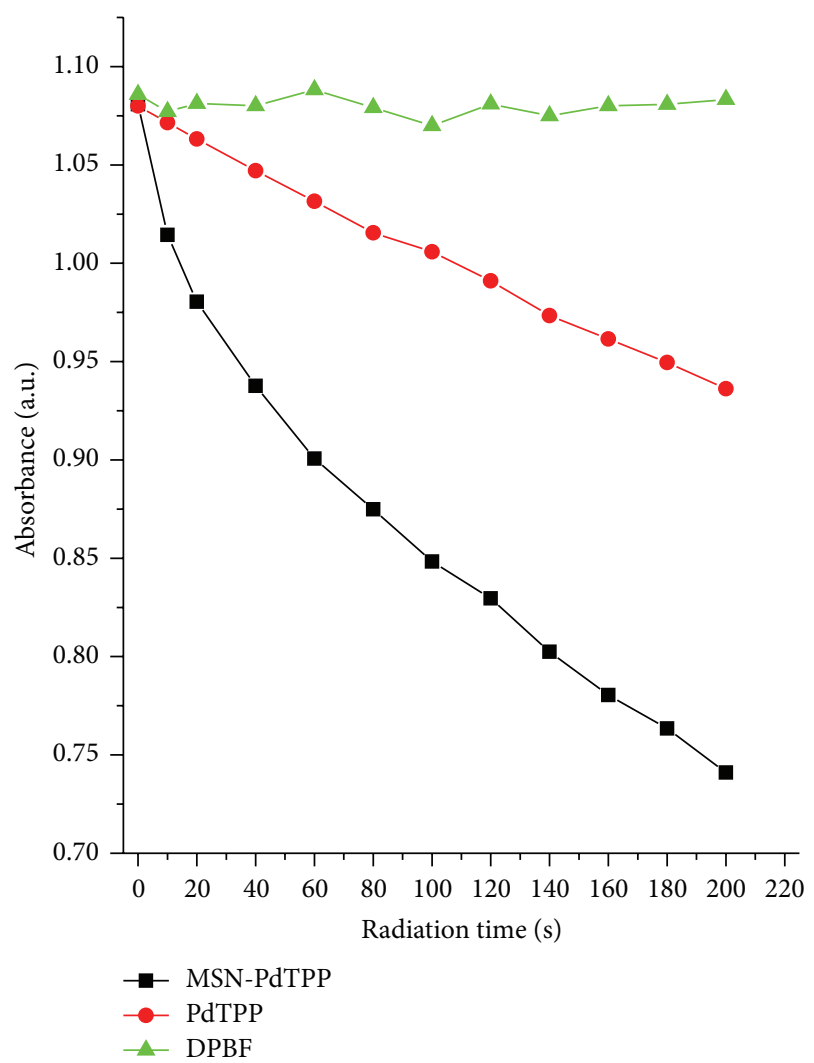

FIGURE 2: Decay curve of DPBF absorbance of ${ }^{1} \mathrm{O}_{2}$ generated from PdTPP and MSN-PdTPP upon photoirradiation. Green line denotes DPBF with photoirradiation (control).

irradiation, respectively. However, significant cytotoxicities were observed for $50 \mu \mathrm{g} \mathrm{m}^{-1}$ MSN-PdTPP at $2.5 \mathrm{~J}$ and $5 \mathrm{~J}$ irradiation (Figure 3(d)). As shown in Figures 3(a) and 3(c), no cytotoxic effects were observed for either free PdTPP or MSN-PdTPP prior to photoirradiation.

With these promising in vitro findings in hand, we then sought to characterize the in vivo utility of our nanoconstructs for PDT by using male $n u / n u$ nude mice bearing MDA-MB-231 xenografts. MDA-MB-231 and MDA-MB-231GFP tumor cells $\left(1 \times 10^{6}\right.$ cells $/ 200 \mu \mathrm{L}$ sterile saline $)$ were injected subcutaneously into the dorsal region of the right and left thigh, respectively (Figure 4). The fluorescent MDAMB-231-GFP tumor cells were inoculated for validation of tumor location and better visualization of growth. After tumors had grown to a volume of approximately $200 \pm$ $50 \mathrm{~mm}^{3}$, we conducted comparative PDT efficacy studies by first dividing animals into four groups $(n=12)$, so as to minimize intergroup weight and tumor size variation. Next two control groups (with/without photoirradiation) were intratumorally injected with $120 \mu \mathrm{L}$ sterile saline solution $(0.9 \% \mathrm{NaCl})$ while the remaining two groups were intratumorally injected with either $50 \mu \mathrm{g}$ of MSN-PdTPP or the same PdTPP-concentration of free PdTPP. One hour after administration, one control group and both free PdTPP and MSN-PdTPP experimental groups were anesthetized and their tumors irradiated at $532 \mathrm{~nm}$ for total energy deposition 


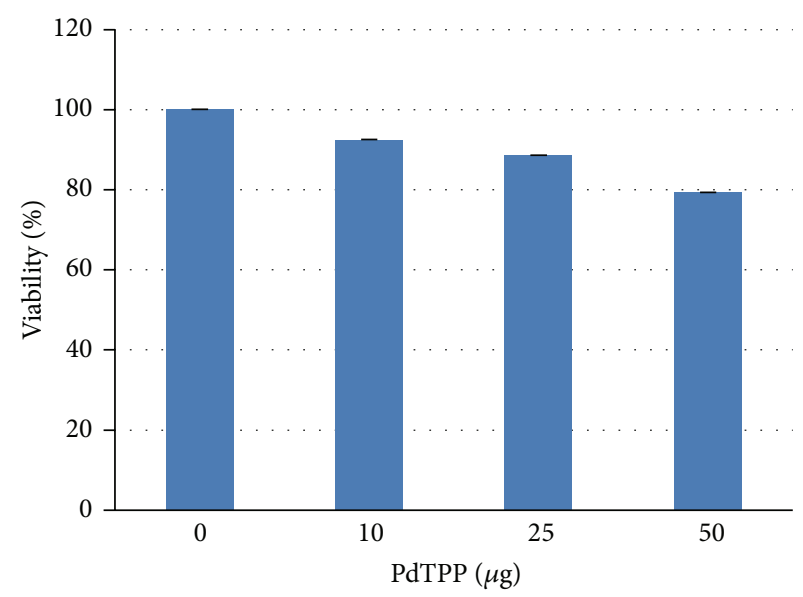

(a) Cytotoxicity of PdTPP

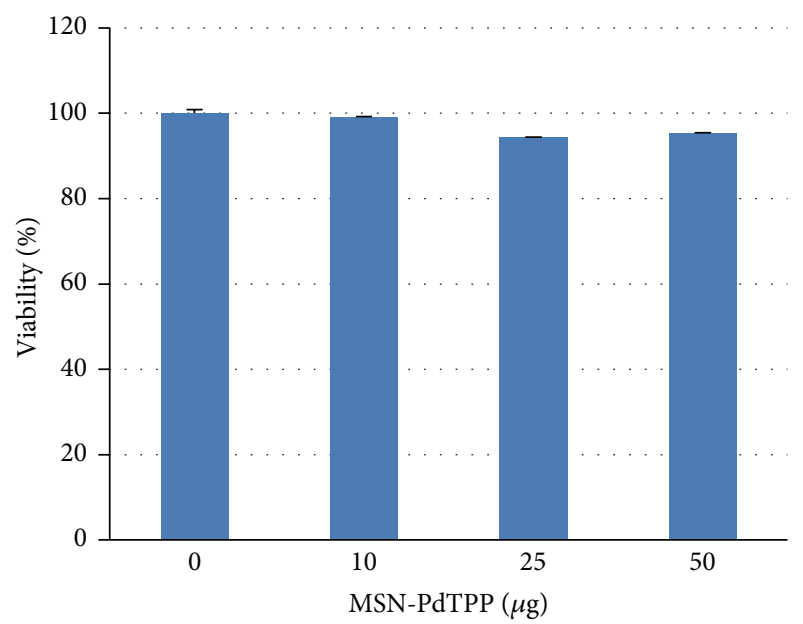

(c) Cytotoxicity of MSN-PdTPP

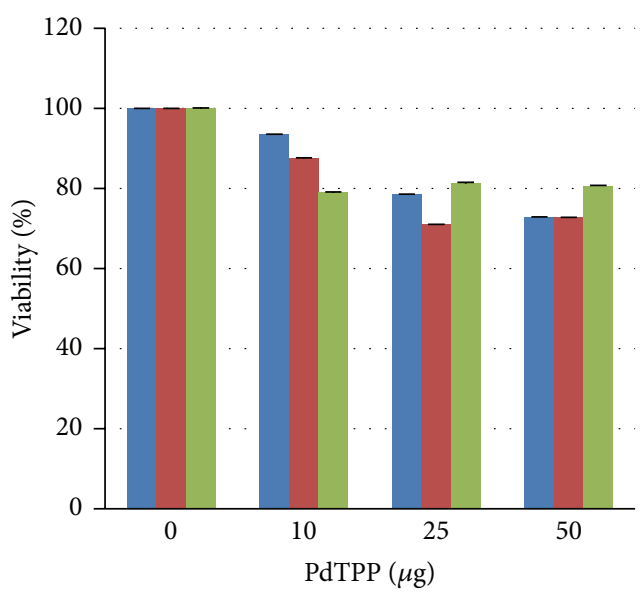

$1.2 \mathrm{~J}$

$2.5 \mathrm{~J}$

$5 \mathrm{~J}$

(b) Cytotoxicity of PdTPP + irradiation

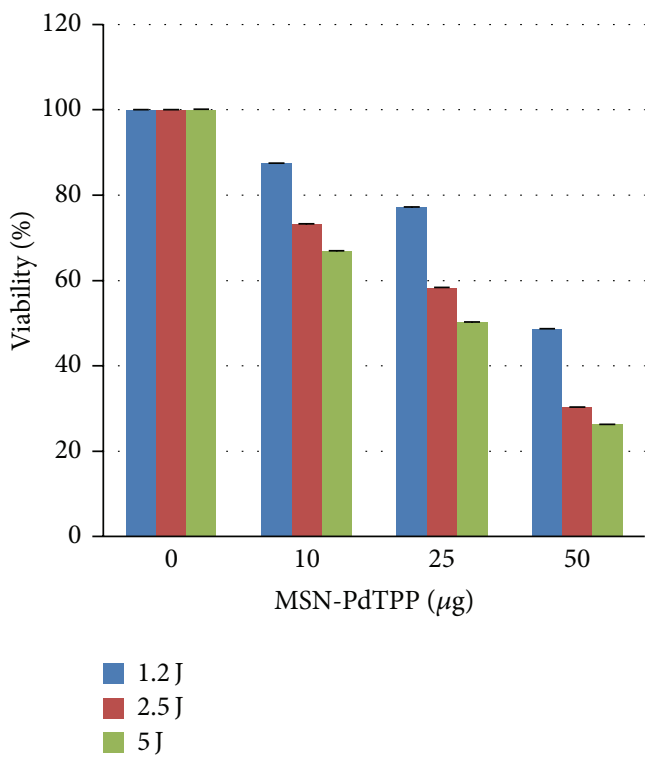

(d) Cytotoxicity of MSN-PdTPP + irradiation

Figure 3: Dark (nonilluminated) cytotoxicity of MDA-MB-231 cells treated with various concentrations of (a) PdTPP and (c) MSN-PdTPP. Illumination cytotoxicity of MDA-MB-231 cells treated with various concentrations of (b) PdTPP and (d) MSN-PdTPP following 1.2 J, 2.5 J, and $5 \mathrm{~J}$ of photoirradiation.

of $80 \mathrm{~J}$. Tumor size and body weight were subsequently monitored on a daily basis, for the next 28 days. As illustrated in Figure 5(a), a single intratumoral administration of MSN-PdTPP and exposure to a single-shot photoirradiation were significantly more efficacious in tumor volume reduction than either single-shot photoirradiated free PdTPP or saline/control (Figure 5(a)). We postulate that the observed marked increase in PDT efficacy afforded by MSN protected delivery of the PS arose primarily from nanoparticleenhanced endocytosis and consequent retention of the PScombining to delay PS clearance from the tumor site as well as to avoid environmental degradation.
Twenty-eight days after PDT treatment, mice were sacrificed and the tumors were removed for measurement (Figure 5(b)). No differences in the long-term PDT benefit were found between the 2 saline control groups (with/without photoirradiation) and the free PdTPP experimental groups (Figure 5(a)), with mean tumor volumes, at the study's end, measuring $10280 \pm 1648 \mathrm{~mm}^{3}$ (saline, no irradiation, $n=$ 12), $9850 \pm 525 \mathrm{~mm}^{3}$ (saline, with irradiation, $n=12$ ), and $10160 \pm 656 \mathrm{~mm}^{3}$ (free PdTPP, with irradiation, $n=12$ ). After intratumoral injection, free PdTPP molecules had the propensity to drain away from the tumor and thus imposed nonobservable PDT effect on tumor following localized 


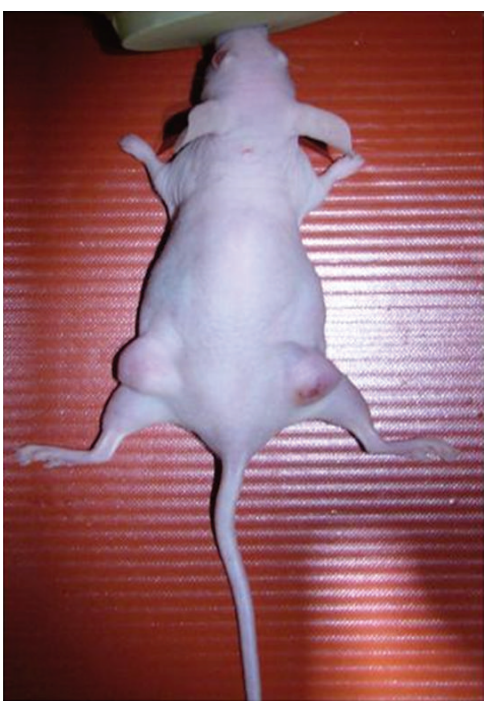

(a)

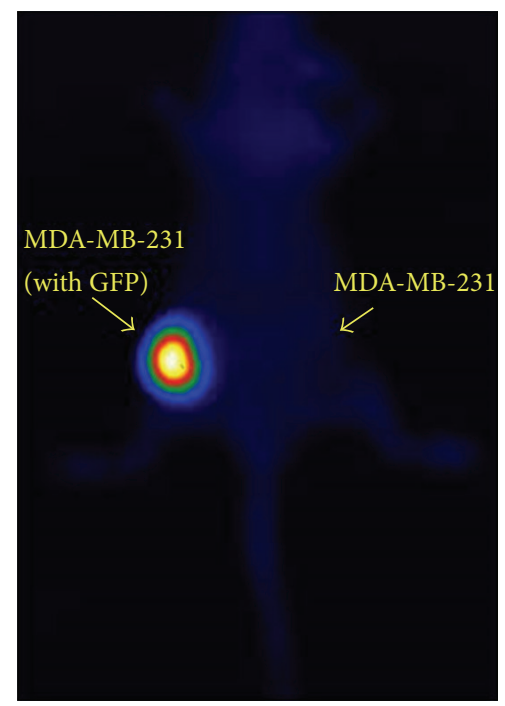

(b)

FIGURE 4: White light (a) and fluorescence (b) images of nude mouse bearing xenografts comprised of GFP-expressing MDA-MB-231 cells on animal's left thigh and nonfluorescent MDA-MB-231 cells on animal's right thigh.

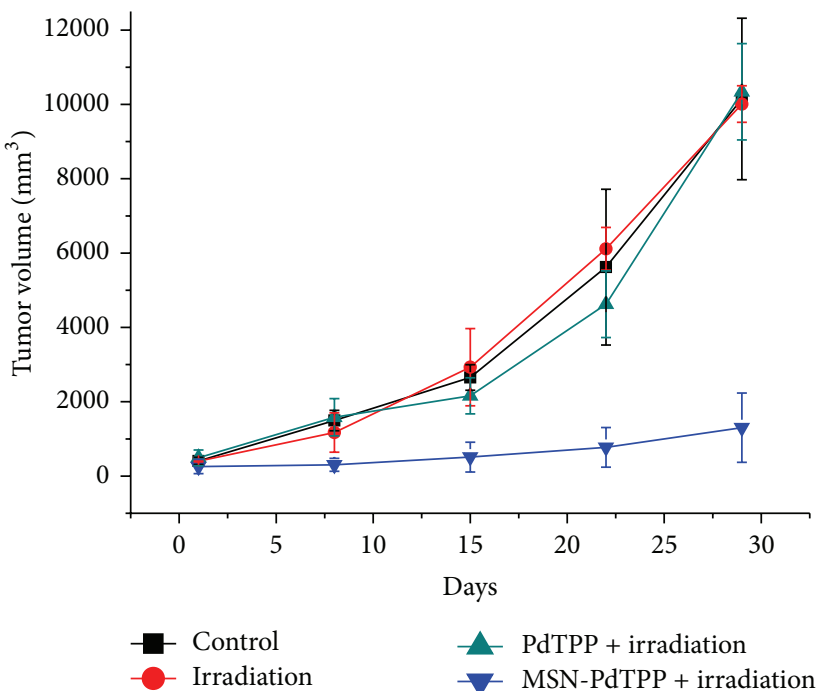

(a)

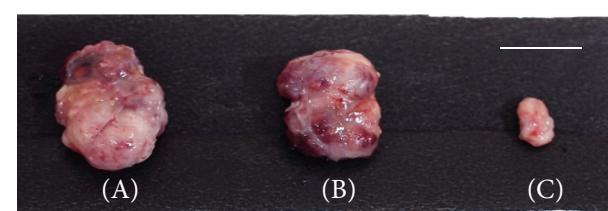

(b)

FIGURE 5: (a) 29-day comparative PDT efficacy studies of MDA-MB-231 s.c. xenograft nude mouse model subjected to a single intratumoral injection of (i) saline without photoirradiation (black squares), (ii) saline with photoirradiation (red circles), (iii) PdTPP with photoirradiation (green upward-pointing triangles), and (iv) MSN-PdTPP with photoirradiation (blue downward-pointing triangles) - showing MSN-PdTPP to be highly efficacious in tumor reduction. Datapoints denote mean of twelve mice per group. (b) Photographs of tumors harvested from mice 28 days after treatment with (A) saline (control), (B) PdTPP, and (C) MSN-PdTPP, all subjected to photoirradiation (scale bar: $1 \mathrm{~cm}$ ).

irradiation. The MSN-PdTPP group, however, demonstrated dramatic PDT benefit at 28 days after irradiation, with a mean tumor volume of $780 \pm 567 \mathrm{~mm}^{3}(n=12)$ as can also be seen from a representative specimen in Figure 5. By contrast, the MSN-PdTPP group without irradiation demonstrated no significant PDT effect, whose tumor volume averagely increased 6-fold 15 days after injection and 13.5-fold 28 days after injection, respectively $(n=5)$. It illustrated a similar tumor growth curve to that of the saline control group.
We then performed hematoxylin and eosin (H\&E) histological staining of excised tumor and injection site tissue sections, confirming the existence of extravasated blood that is characteristic of PDT (Figure 6(a)). To more precisely assess antitumor response to our nanoparticle PDT treatments, we compared terminal deoxynucleotidyl transferase dUTP nick end labeling (TUNEL) and caspase-3 immunohistochemical staining for apoptosis of tumors harvested from mice that had been treated with MSN-PdTPP nanoparticles for $0 \mathrm{~h}$ and $1 \mathrm{~h}$ 

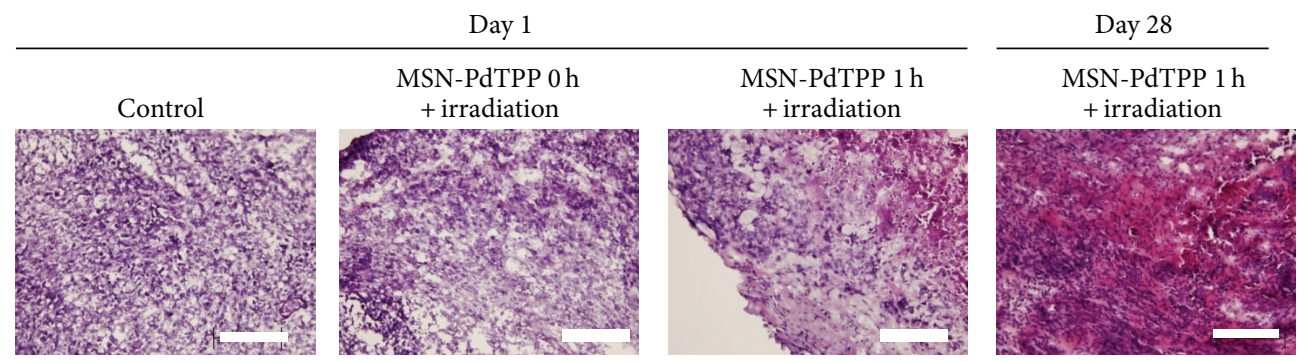

(a) $H \& E$
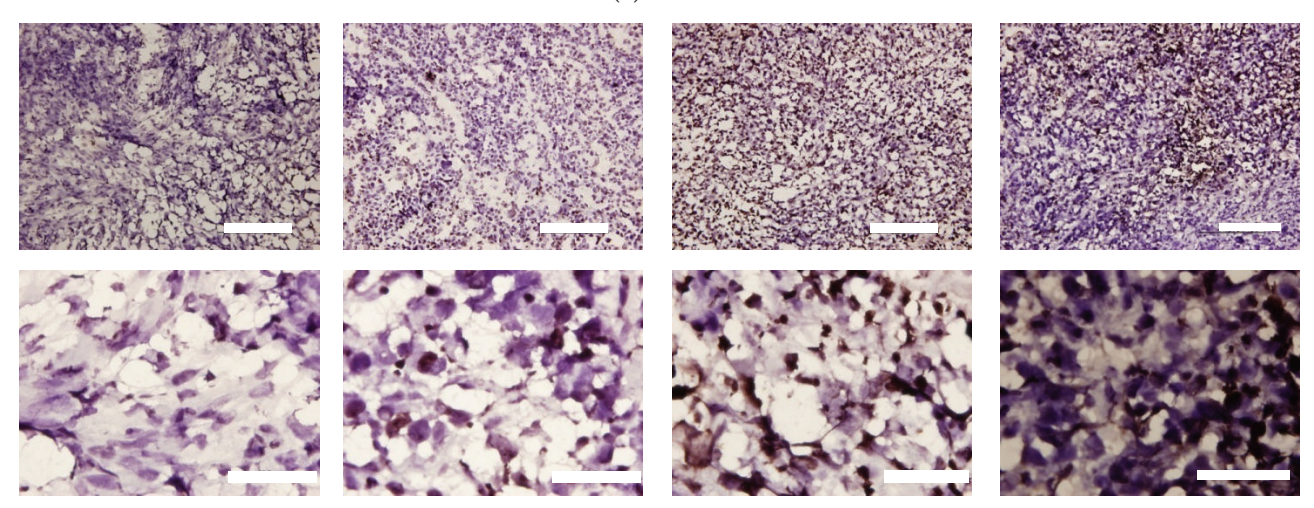

(b) TUNEL
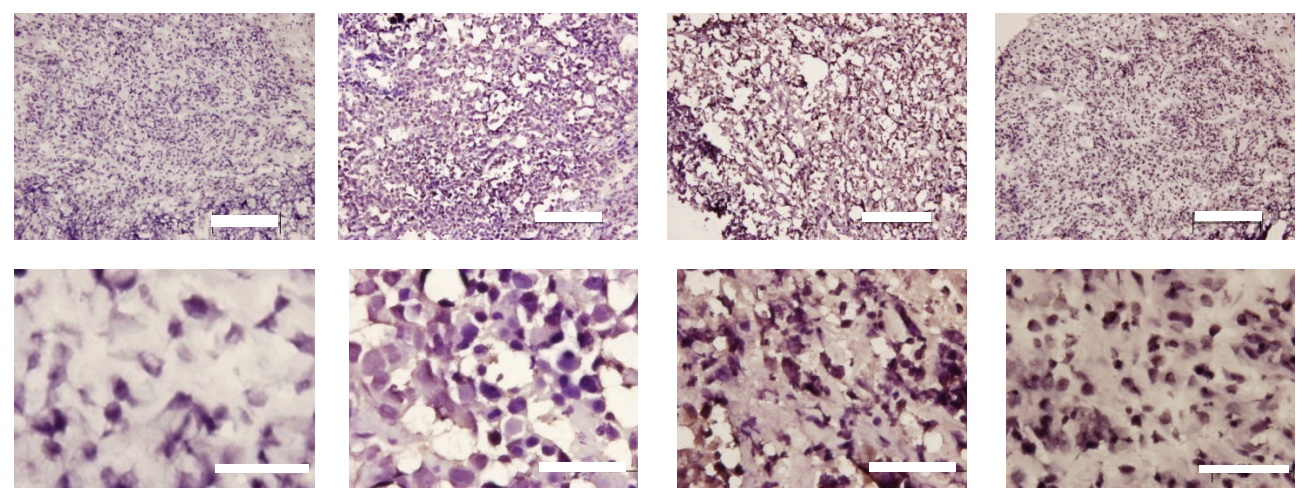

(c) Caspase-3

FIGURE 6: (a) Histological staining of the excised tumor sections on treatment day 1 of (i) saline with no photoirradiation (control), (ii) MSNPdTPP with photoirradiation at $0 \mathrm{~h}$ after injection, (iii) MSN-PdTPP with photoirradiation at $1 \mathrm{~h}$ after injection, and on treatment day 28 of (iv) MSN-PdTPP with photoirradiation at $1 \mathrm{~h}$ after injection. H\&E staining; scale bars: $200 \mu \mathrm{m}$. (b) TUNEL staining of consecutive sections for each group at 200x magnification (upper, scale bars: $200 \mu \mathrm{m}$ ) and 1000x magnification (upper, scale bars: $50 \mu \mathrm{m}$ ). (c) Caspase-3 staining of consecutive sections for each group at 200x magnification (upper, scale bars: $200 \mu \mathrm{m}$ ) and 1000x magnification (upper, scale bars: $50 \mu \mathrm{m}$ ).

with irradiation, as well as from negative controls (Figures 6(b) and 6(c)). Tumors treated with MSN-PdTPP for $1 \mathrm{~h}$ demonstrated considerable apoptosis one day following their photoirradiation. Stained sections of MSN-PdTPP-treated tumors harvested 28 days after irradiation revealed much greater apoptosis still. By contrast, TUNEL and caspase-3 staining of samples that had been immediately photoirradiated following MSN-PdTPP administration revealed little apoptosis. These findings suggested that, even with MSNPdTPP's appreciable generation of ${ }^{1} \mathrm{O}_{2}$, PDT therapeutic benefit strongly depended upon efficient nanoplatform endocytosis.
To further characterize our nanoparticle's uptake by cancer cells in vivo, we conducted TEM of MDA-MB-231 tumor sections harvested 0,1 , and $6 \mathrm{~h}$ after intratumoral injection (Figure 7). As shown in Figures 7(a) and 7(b), the TEM images of tumors treated $1 \mathrm{~h}$ prior with MSN-PdTPP demonstrated greater nanoparticle uptake than those harvested immediately following nanoparticle administration, with nanoparticles mostly confined to the periphery of cells at $0 \mathrm{~h}$ and not yet significantly endocytosed. TEM images of tumors treated $6 \mathrm{~h}$ prior, however, revealed few nanoparticles remaining within harvested tumors. Taken together we posit that optimal endocytosis and thus optimal photoirradiation 

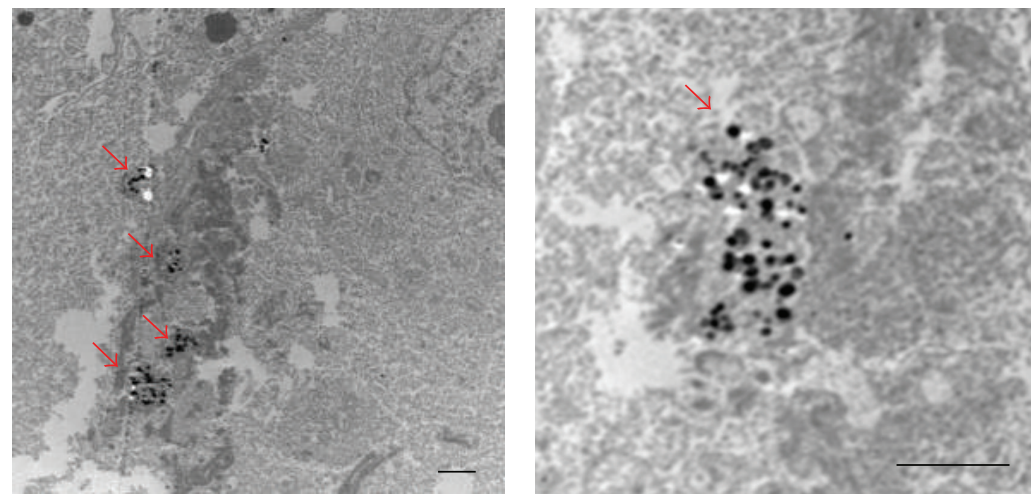

(a)
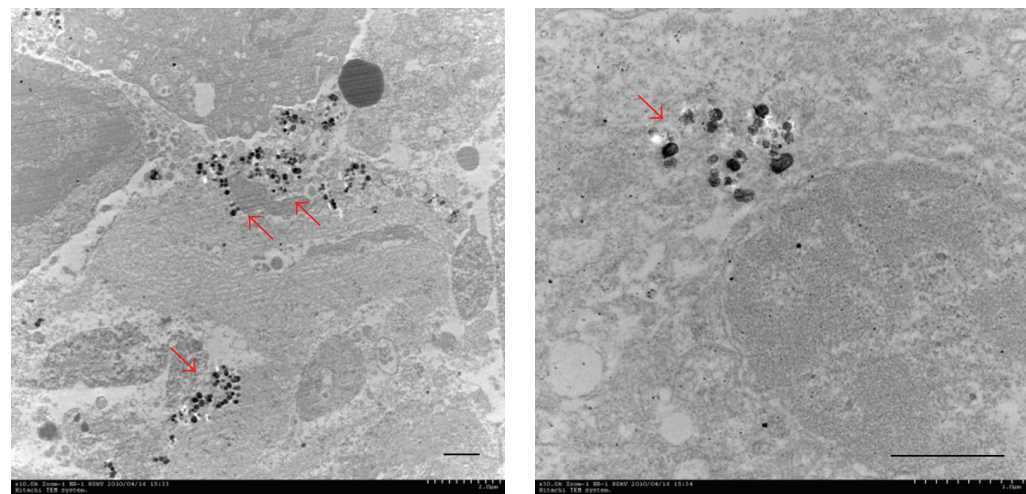

(b)
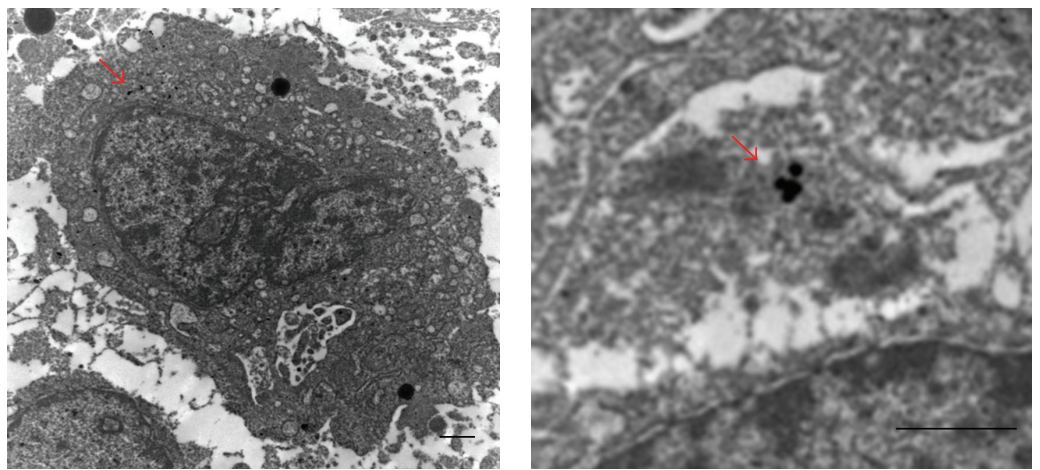

(c)

FIGURE 7: TEM images of tissue sections of tumors harvested at (a) $0 \mathrm{~h},(\mathrm{~b}) 1 \mathrm{~h}$, and (c) $6 \mathrm{~h}$ following intratumoral injection of MSN-PdTPP nanoparticles. Red arrows indicate MSN-PdTPP nanoparticle accumulations (scale bars: $1 \mu \mathrm{m}$ ).

are likely to be somewhere between 1 and $6 \mathrm{~h}$ following intratumoral injection of our nanoparticles.

\section{Conclusions}

In this work we describe significant enhancement of photodynamic therapy efficacy achieved via the use of nanoparticlebased photosensitizers, even when treatment is limited to a single photosensitizer dose and a single photoirradiation session. By covalently conjugating large numbers of the photosensitizer Pd-porphyrin onto the environmentally communicative nanochannel walls of mesoporous silica nanoparticles, we circumvent our hydrophobic photosensitizer's tendency to self-aggregate and self-quench. The benefits of this protected conveyance of substantial quantities of photosensitizer, our nanoplatform's proclivity for endocytosis, and thereby photosensitizer accumulation within tumor permit the use of much lower photosensitizer dose than free photosensitizer and with much greater antitumor response. Current efforts are aimed at the surface functionalization of 
our nanoplatform for pathology-specific targeting and further optimization of pathology-dependent timing/frequency of nanoparticle dose and photoirradiation.

\section{Conflict of Interests}

The authors declare that there is no conflict of interests regarding the publication of this paper.

\section{Acknowledgments}

The authors acknowledge financial support from the National Health Research Institutes of Taiwan (BN-103-PP-04 and NM-103-PP-01) and the National Science Council of Taiwan (NSC-100-2911-I-400-502 and NSC102-2113-M-400-001MY3).

\section{References}

[1] D. E. J. G. J. Dolmans, D. Fukumura, and R. K. Jain, "Photodynamic therapy for cancer," Nature Reviews Cancer, vol. 3, no. 5, pp. 380-387, 2003.

[2] T. J. Dougherty, C. J. Gomer, G. Jori et al., "Photodynamic therapy," Journal of the National Cancer Institute, vol. 90, no. 12, pp. 889-905, 1998.

[3] R. R. Allison, V. S. Bagnato, and C. H. Sibata, "Future of oncologic photodynamic therapy," Future Oncology, vol. 6, no. 6, pp. 929-940, 2010.

[4] C. M. Moore, D. Pendse, and M. Emberton, "Photodynamic therapy for prostate cancer-a review of current status and future promise," Nature Clinical Practice Urology, vol. 6, no. 1, pp. 18-30, 2009.

[5] A. E. O'Connor, W. M. Gallagher, and A. T. Byrne, "Porphyrin and nonporphyrin photosensitizers in oncology: preclinical and clinical advances in photodynamic therapy," Photochemistry and Photobiology, vol. 85, no. 5, pp. 1053-1074, 2009.

[6] J. Saczko, A. Chwiłkowska, J. Kulbacka et al., "Photooxidative action in cancer and normal cells induced by the use of photofrin in photodynamic therapy," Folia Biologica, vol. 54, no. 1, pp. 24-29, 2008.

[7] M. A. El-Sayed, "The triplet state: Its radiative and nonradiative properties," Accounts of Chemical Research, vol. 1, no. 1, pp. 8-16, 1968.

[8] S. P. McGlynn, T. Azumi, and M. Kinoshita, Molecular Spectroscopy of the Triplet State, Prentice Hall, Englewood Cliffs, NJ, USA, 1969.

[9] M. C. DeRosa and R. J. Crutchey, "Photosensitized singlet oxygen and its applications," Coordination Chemistry Reviews, vol. 223-234, pp. 351-371, 2002.

[10] Z. Luksiene, "Photodynamic therapy: mechanism of action and ways to improve the efficiency of treatment," Medicina, vol. 39, no. 12, pp. 1137-1150, 2003.

[11] I. J. MacDonald and T. J. Dougherty, "Basic principles of photodynamic therapy," Journal of Porphyrins and Phthalocyanines, vol. 5, no. 2, pp. 105-129, 2001.

[12] D. K. Chatterjee, L. S. Fong, and Y. Zhang, "Nanoparticles in photodynamic therapy: an emerging paradigm," Advanced Drug Delivery Reviews, vol. 60, no. 15, pp. 1627-1637, 2008.

[13] D. Bechet, P. Couleaud, C. Frochot, M. Viriot, F. Guillemin, and M. Barberi-Heyob, "Nanoparticles as vehicles for delivery of photodynamic therapy agents," Trends in Biotechnology, vol. 26, no. 11, pp. 612-621, 2008.

[14] W. C. Zamboni, V. Torchilin, A. K. Patri et al., "Best practices in cancer nanotechnology: perspective from NCI nanotechnology alliance," Clinical Cancer Research, vol. 18, no. 12, pp. 3229-3241, 2012.

[15] L. Zhang, F. X. Gu, J. M. Chan, A. Z. Wang, R. S. Langer, and O. C. Farokhzad, "Nanoparticles in medicine: therapeutic applications and developments," Clinical Pharmacology and Therapeutics, vol. 83, no. 5, pp. 761-769, 2008.

[16] R. Tong and D. S. Kohane, "Shedding light on nanomedicine," WIREs Nanomed Nanobiotechnol, vol. 4, no. 6, pp. 638-662, 2012.

[17] C. Lim, J. Heo, S. Shin et al., "Nanophotosensitizers toward advanced photodynamic therapy of cancer," Cancer Letters, vol. 334, no. 2, pp. 176-187, 2013.

[18] E. Paszko, C. Ehrhardt, M. O. Senge, D. P. Kelleher, and J. V. Reynolds, "Nanodrug applications in photodynamic therapy," Photodiagnosis and Photodynamic Therapy, vol. 8, no. 1, pp. 1429, 2011.

[19] S. H. Cheng and L. W. Lo, "Inorganic nanoparticles for enhanced photodynamic cancer therapy," Current Drug Discovery Technologies, vol. 8, no. 3, pp. 250-268, 2011.

[20] J. M. Tsay, M. Trzoss, L. Shi et al., "Singlet oxygen production by peptide-coated quantum dot-photosensitizer conjugates," Journal of the American Chemical Society, vol. 129, no. 21, pp. 6865-6871, 2007.

[21] Y. Liu, W. Chen, S. Wang, and A. G. Joly, "Investigation of watersoluble $\mathrm{x}$-ray luminescence nanoparticles for photodynamic activation," Applied Physics Letters, vol. 92, no. 4, Article ID 043901, 2008.

[22] D. K. Chatterjee and Z. Yong, "Upconverting nanoparticles as nanotransducers for photodynamic therapy in cancer cells," Nanomedicine, vol. 3, no. 1, pp. 73-82, 2008.

[23] J. P. Celli, B. Q. Spring, I. Rizvi et al., "Imaging and photodynamic therapy: mechanisms, monitoring, and optimization," Chemical Reviews, vol. 110, no. 5, pp. 2795-2838, 2010.

[24] S. H. Cheng, C. C. Hsieh, N. T. Chen et al., "Well-defined mesoporous nanostructure modulates three-dimensional interface energy transfer for two-photon activated photodynamic therapy," Nano Today, vol. 6, no. 6, pp. 552-563, 2011.

[25] M. Gary-Bobo, Y. Mir, C. Rouxel et al., "Mannose-functionalized mesoporous silica nanoparticles for efficient two-photon photodynamic therapy of solid tumors," Angewandte Chemie International Edition, vol. 50, no. 48, pp. 11425-11429, 2011.

[26] C. Liu, S. Cheng, N. Chen, and L. Lo, "Intra/inter-particle energy transfer of luminescence nanocrystals for biomedical applications," Journal of Nanomaterials, vol. 2012, Article ID 706134, 9 pages, 2012.

[27] J. Lu, Z. Li, J. I. Zink, and F. Tamanoi, "In vivo tumor suppression efficacy of mesoporous silica nanoparticles-based drug-delivery system: enhanced efficacy by folate modification," Nanomedicine, vol. 8, no. 2, pp. 212-220, 2012.

[28] L. Li, F. Tang, H. Liu et al., "In vivo delivery of silica nanorattle encapsulated docetaxel for liver cancer therapy with low toxicity and high efficacy," ACS Nano, vol. 4, no. 11, pp. 6874-6882, 2010.

[29] Y. Cao, R. A. Depinho, M. Ernst, and K. Vousden, "Cancer research: past, present and future," Nature Reviews Cancer, vol. 11, no. 10, pp. 749-754, 2011. 
[30] V. Mamaeva, J. M. Rosenholm, L. T. Bate-Eya et al., “Mesoporous silica nanoparticles as drug delivery systems for targeted inhibition of notch signaling in cancer," Molecular Therapy, vol. 19, no. 8, pp. 1538-1546, 2011.

[31] H. Meng, M. Xue, T. Xia et al., "Use of size and a copolymer design feature to improve the biodistribution and the enhanced permeability and retention effect of doxorubicin-loaded mesoporous silica nanoparticles in a murine xenograft tumor model," ACS Nano, vol. 5, no. 5, pp. 4131-4144, 2011.

[32] J. Lu, M. Liong, Z. Li, J. I. Zink, and F. Tamanoi, "Biocompatibility, biodistribution, and drug-delivery efficiency of mesoporous silica nanoparticles for cancer therapy in animals," Small, vol. 6, no. 16, pp. 1794-1805, 2010.

[33] J. E. Lee, N. Lee, T. Kim, J. Kim, and T. Hyeon, "Multifunctional mesoporous silica nanocomposite nanoparticles for theranostic applications," Accounts of Chemical Research, vol. 44, no. 10, pp. 893-902, 2011.

[34] Z. Li, J. C. Barnes, A. Bosoy, J. F. Stoddart, and J. I. Zink, "Mesoporous silica nanoparticles in biomedical applications," Chemical Society Reviews, vol. 41, no. 7, pp. 2590-2605, 2012.

[35] V. Mamaeva, C. Sahlgren, and M. Lindén, "Mesoporous silica nanoparticles in medicine-recent advances," Advanced Drug Delivery Reviews, vol. 65, no. 5, pp. 689-702, 2012.

[36] Y. W. Yang, "Towards biocompatible nanovalves based on mesoporous silica nanoparticles," MedChemComm, vol. 2, no. 11, pp. 1033-1049, 2011.

[37] S. Wu, Y. Hung, and C. Mou, "Mesoporous silica nanoparticles as nanocarriers," Chemical Communications, vol. 47, no. 36, pp. 9972-9985, 2011.

[38] F. Tang, L. Li, and D. Chen, "Mesoporous silica nanoparticles: synthesis, biocompatibility and drug delivery," Advanced Materials, vol. 24, no. 12, pp. 1504-1534, 2012.

[39] W. Huang and J. J. Davis, "Multimodality and nanoparticles in medical imaging," Dalton Transactions, vol. 40, no. 23, pp. 60876103, 2011.

[40] S. H. Cheng, C. H. Lee, M. C. Chen et al., "Tri-functionalization of mesoporous silica nanoparticles for comprehensive cancer theranostics: the trio of imaging, targeting and therapy," Journal of Materials Chemistry, vol. 20, no. 29, pp. 6149-6157, 2010.

[41] S. Cheng, C. Lee, C. Yang, F. Tseng, C. Mou, and L. Lo, "Mesoporous silica nanoparticles functionalized with an oxygensensing probe for cell photodynamic therapy: potential cancer theranostics," Journal of Materials Chemistry, vol. 19, no. 9, pp. 1252-1257, 2009.

[42] H.-L. Tu, Y.-S. Lin, H.-Y. Lin et al., "In vitro studies of functionalized mesoporous silica nanoparticles for photodynamic therapy," Advanced Materials, vol. 21, no. 2, pp. 172-177, 2009. 

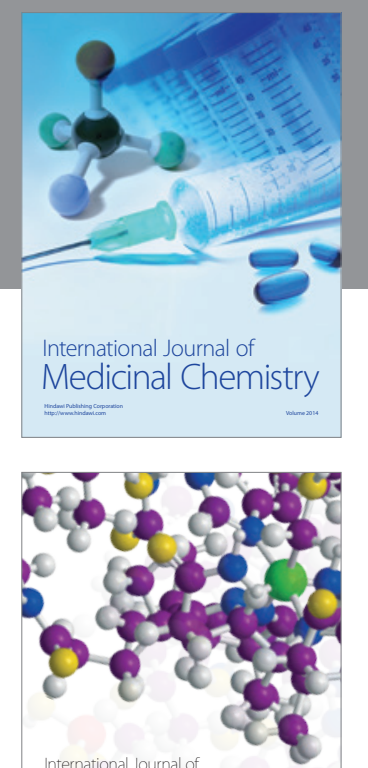

\section{Carbohydrate} Chemistry

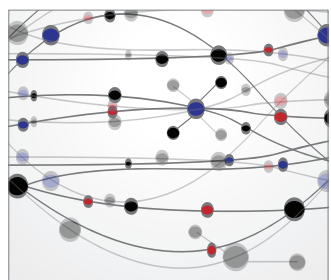

The Scientific World Journal
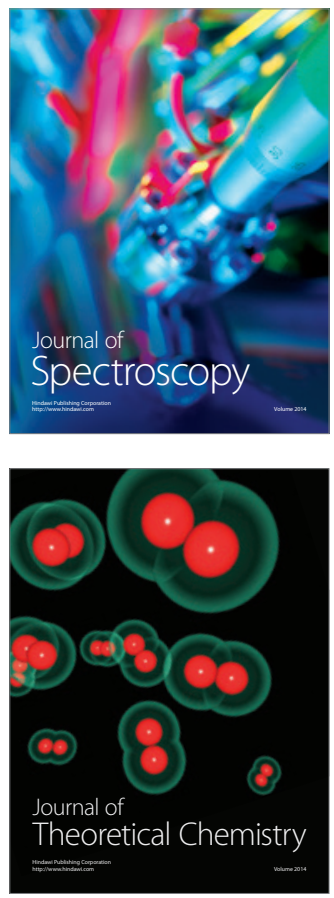
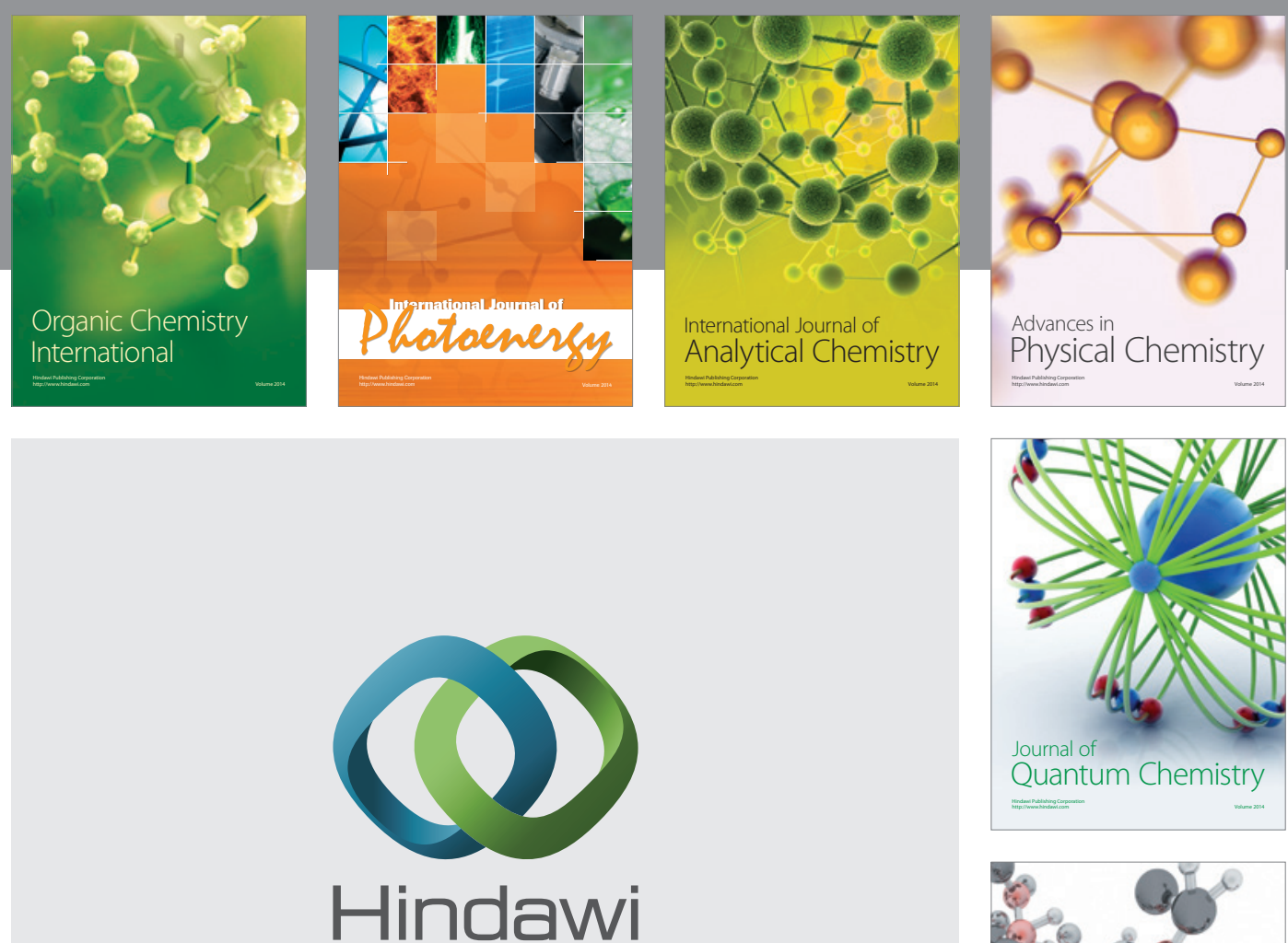

Submit your manuscripts at

http://www.hindawi.com

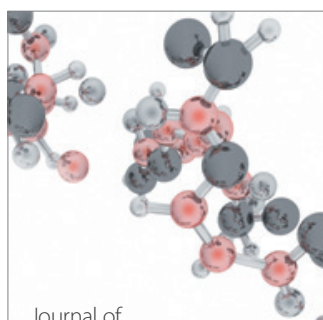

Analytical Methods

in Chemistry

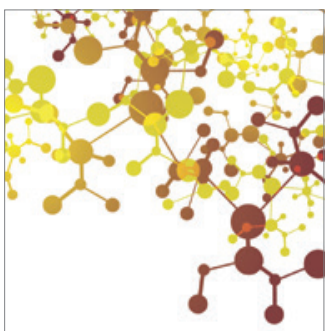

Journal of

Applied Chemistry

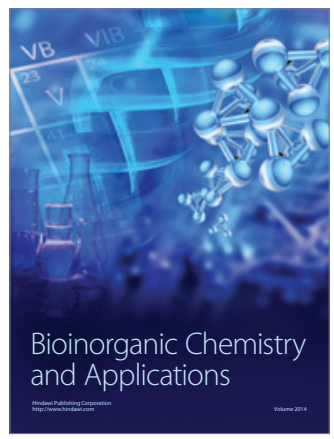

Inorganic Chemistry
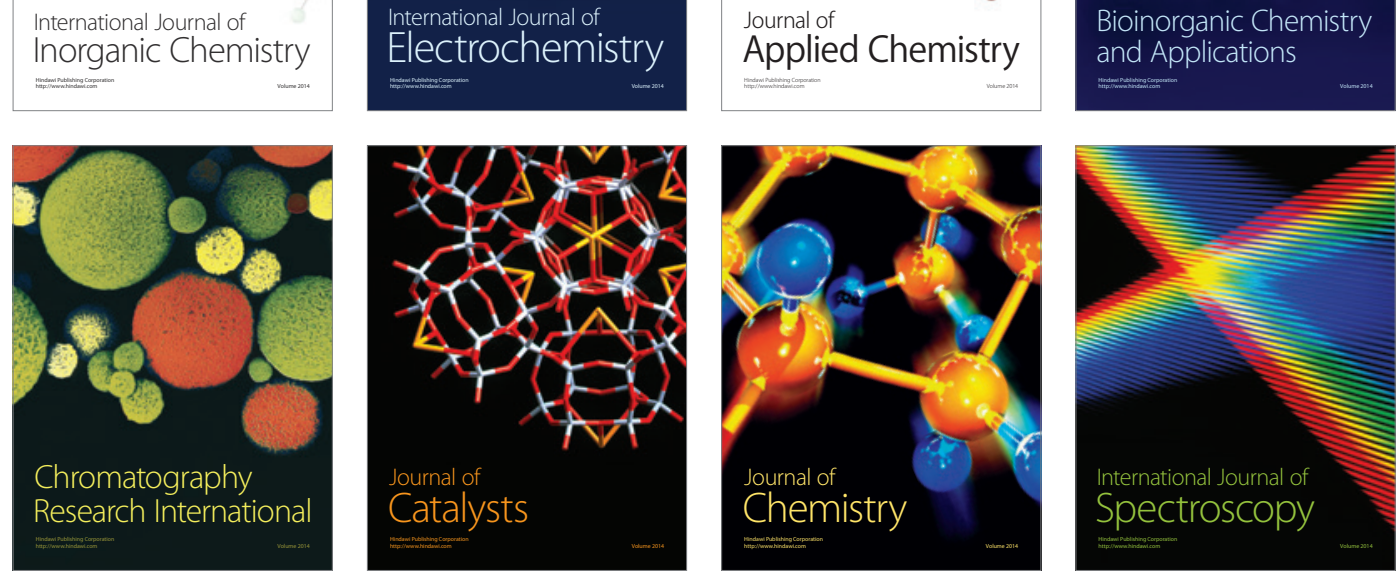